\title{
Predicting mortality during long-term follow-up in pulmonary arterial hypertension
}

\section{David Kylhammar ${ }^{1}$, Clara Hjalmarsson (1) ${ }^{2}$, Roger Hesselstrand ${ }^{3}$, Kjell Jansson ${ }^{1}$, Mohammad Kavianipour ${ }^{4}$, Barbro Kjellström (1) 5,6, Magnus Nisell ${ }^{7}$, Stefan Söderberg $\mathbb{1}^{8}$ and Göran Rådegran ${ }^{9}$ on behalf of the Swedish Association for Pulmonary Hypertension (SveFPH) and the Swedish Pulmonary Arterial Hypertension Registry (SPAHR)}

\begin{abstract}
Affiliations: 'Division of Diagnostics and Specialist Medicine, Dept of Health, Medicine and Caring Sciences, and Dept of Clinical Physiology, Linköping University, Linköping, Sweden. ${ }^{2}$ Department of Molecular and Clinical Medicine, Sahlgrenska Academy, Gothenburg University, and Dept of Cardiology, Sahlgrenska University Hospital, Gothenburg, Sweden. ${ }^{3}$ Dept of Clinical Sciences Lund, Rheumatology, Lund University, and Skåne University Hospital, Lund, Sweden. ${ }^{4}$ Dept of Public Health and Clinical Medicine, Sundsvall Research Unit, Umeå University, Umeå, Sweden. ${ }^{5}$ Dept of Clinical Sciences in Lund, Clinical Physiology, Faculty of Medicine, Lund University, and Skåne University Hospital, Lund, Sweden. ${ }^{6}$ Cardiology Unit, Dept of Medicine, Karolinska Institute, Stockholm, Sweden. ${ }^{7}$ Lung Unit, Dept of Medicine, Karolinska Institute, and Karolinska University Hospital, Stockholm, Sweden. ${ }^{8}$ Dept of Public Health and Clinical Medicine, and Heart Centre, Umeå University, Umeå, Sweden. ${ }^{9}$ Dept of Clinical Sciences Lund, Cardiology, Lund University, and Skåne University Hospital, Lund, Sweden.
\end{abstract}

Correspondence: David Kylhammar, Dept of Clinical Physiology, Linköping University Hospital, SE-581 85 , Linköping, Sweden. E-mail: david.kylhammaraliu.se

ABSTRACT The European Society of Cardiology (ESC) and European Respiratory Society (ERS) guideline recommendation of comprehensive risk assessments, which classify patients with pulmonary arterial hypertension (PAH) as having low, intermediate or high mortality risk, has not been evaluated during long-term follow-up in a "real-life" clinical setting. We therefore aimed to investigate the utility of risk assessment in a clinical setting for up to 5 years post diagnosis.

386 patients with PAH from the Swedish PAH Registry were included. Risk group (low/intermediate/ high) and proportion of low-risk variables were investigated at 3-, 4- and 5-year follow-ups after time of diagnosis. In an exploratory analysis, survival rates of patients with low-intermediate or high-intermediate risk scores were compared.

A low-risk profile was in multivariate Cox proportional hazards regressions found to be a strong, independent predictor of longer transplant-free survival $(\mathrm{p}<0.001)$ at the 3 -, 4 - and 5 -year follow-ups. Also, for the 3-, 4- and 5-year follow-ups, survival rates significantly differed $(\mathrm{p}<0.001)$ between the three risk groups. Patients with a greater proportion of low-risk variables had better $(\mathrm{p}<0.001)$ survival rates. Patients with a high-intermediate risk score had worse survival rates $(\mathrm{p}<0.001)$ than those with a lowintermediate risk score. Results were similar when excluding patients with $\geqslant 3$ risk factors for heart failure with preserved ejection fraction, atrial fibrillation and/or age $>75$ years at diagnosis.

Our findings suggest that the ESC/ERS guideline strategy for comprehensive risk assessments in PAH is valid also during long-term follow-up in a "real-life" clinical setting.

@ERSpublications

ESC/ERS guidelines' risk stratification table successfully predicts outcome during long-term follow-up in pulmonary arterial hypertension https://bit.ly/3nXJbop

Cite this article as: Kylhammar D, Hjalmarsson C, Hesselstrand R, et al. Predicting mortality during long-term follow-up in pulmonary arterial hypertension. ERJ Open Res 2021; 7: 00837-2020 [https:// doi.org/10.1183/23120541.00837-2020].

This article has supplementary material available from openres.ersjournals.com

Received: 10 Nov 2020 | Accepted after revision: 6 Jan 2021

Copyright $\odot$ The authors 2021. This version is distributed under the terms of the Creative Commons Attribution NonCommercial Licence 4.0. For commercial reproduction rights and permissions contact permissions@ersnet.org 


\section{Introduction}

Pulmonary arterial hypertension (PAH) is a severe, progressive pulmonary vascular disease associated with significant morbidity and mortality [1-3]. The disease can be idiopathic, hereditary or associated with other conditions, most frequently connective tissue or congenital heart diseases $[4,5]$.

In order to optimise patient care, treatment and outcome, frequent monitoring is recommended with the consensus that comprehensive risk assessments should be performed at diagnosis and during follow-up. The European Society of Cardiology (ESC) and European Respiratory Society (ERS) guidelines from 2015 suggest that a specific "risk table", classifying patients as low, intermediate or high risk, should be used to estimate mortality in patients with $\mathrm{PAH}$ [6]. This approach was later retrospectively validated at time of diagnosis and at an early follow-up, within a maximum of 1-2 years from diagnosis. Three abbreviated risk stratification models, based on the ESC/ERS "risk table", were then applied to data from incident (i.e. newly diagnosed) patients with PAH included in the Swedish [7], French [8] and COMPERA [9] PAH registries.

The use of the "risk table" is, nevertheless, also recommended for later follow-up of disease progression. Importantly, two recent studies performed post hoc analyses of the PATENT-1, its open-label extension PATENT-2 [10] and the GRIPHON [11] studies, respectively, and found that the abbreviated versions of the ESC/ERS "risk table" discriminate patients with better or worse outcome, also in mostly prevalent and previously treated patients included in these randomised controlled trials.

The principal aim of this study was, however, to test the utility of the ESC/ERS risk stratification model for assessing mortality risk up to 5 years post diagnosis in a "real-life" population of patients from the Swedish PAH Registry (SPAHR).

\section{Methods}

\section{SPAHR and the study population}

The study was based on data from SPAHR, which was launched in 2008 and includes consecutive data from incident cases of PAH diagnosed thereafter at the PAH centres in Gothenburg, Linköping, Lund, Stockholm, Umeå/Sundsvall, Uppsala and Örebro. Data from retrospectively registered cases diagnosed between 2000 and 2007 are also included. Data registration is manually and voluntarily performed at each centre. SPAHR includes information on demographics, comorbidities (hypertension, diabetes mellitus, atrial fibrillation, previous stroke, ischaemic heart disease and thyroid disease at time of diagnosis), treatments, World Health Organization (WHO) functional class (FC), 6-min walk distance (6MWD), blood biochemistry, and data from echocardiography and right heart catheterisation. SPAHR is approved by the National Board of Health and Welfare and the Swedish Data Inspection. Patients are locally informed about SPAHR participation and have the right to decline. The study complies with the Declaration of Helsinki and was approved by the local ethics committee in Lund (Dnr. 2010-114, Dnr. 2017-531).

All cases classified as PAH group I according to the Nice classification [4, 5] who were not acute vaso-responders and were diagnosed from 2002 to 2015 were included if they had survived $\geqslant 2.5$ years from diagnosis. The exclusion of acute vaso-responders was motivated by their different treatment and prognosis. The diagnosis of PAH was set according to guidelines at that time $[6,12,13]$. Patients were categorised as idiopathic/familial (IPAH/FPAH), connective tissue disease-associated (APAH-CTD), congenital heart disease-associated (APAH-CHD) or other forms of associated PAH (APAH-Others), including drugs- and toxins-induced PAH, HIV-associated PAH and portal hypertension-associated PAH. Registered follow-ups performed after 30-42, 43-54 and 55-66 months from diagnosis were included, representing 3-, 4- and 5 -year follow-ups, respectively. If multiple follow-ups were registered within any of these timeframes, the follow-up with most variables for risk assessment was included. If the number of variables was equal, the earliest follow-up was chosen. A patient could be included at one, two or three follow-ups.

Creatinine levels were used to estimate glomerular filtration rate (eGFR) according to the Cockcroft-Gault formula [14]. Kidney dysfunction was defined as eGFR $<60 \mathrm{~mL} \cdot \mathrm{min}^{-1} \cdot \mathrm{m}^{-2}$. Body mass index (BMI) was calculated as weight/(length $)^{2}$.

\section{Comprehensive risk assessments}

Patients were classified as low-, intermediate- or high risk according to the previously described "SPAHR equation", an adjustment of the ESC/ERS guidelines' risk stratification model to fit a retrospective registry-based data set, and by which the risk group for a specific patient is determined by the use of cut-off values for FC, 6MWD, N-terminal pro-hormone of brain natriuretic peptide, right atrial area, mean right atrial pressure, pericardial effusion, cardiac index and/or mixed venous oxygen saturation, as defined in the "risk table" of the 2015 ESC/ERS guidelines [6] (table 1). Patients in the intermediate risk 


\begin{tabular}{|c|c|c|c|}
\hline Determinants of prognosis & Low risk & Intermediate risk & High risk \\
\hline WHO functional class & I, II & III & IV \\
\hline 6MWD m & $>440$ & $165-440$ & $<165$ \\
\hline NT-proBNP levels $n g \cdot L^{-1}$ & $<300$ & $300-1400$ & $>1400$ \\
\hline Imaging (echocardiography) & $\mathrm{RA}$ area $<18 \mathrm{~cm}^{2}$ & $\mathrm{RA}$ area $18-26 \mathrm{~cm}^{2}$ & $\mathrm{RA}$ area $>26 \mathrm{~cm}^{2}$ \\
\hline & No pericardial effusion & & Pericardial effusion \\
\hline Haemodynamics & & & \\
\hline $\mathrm{RAP} \mathrm{mmHg}$ & $<8$ & $8-14$ & $>14$ \\
\hline $\mathrm{Cl} L \cdot \mathrm{min}^{-1} \cdot \mathrm{m}^{-2}$ & $\geqslant 2.5$ & $2.0-2.4$ & $<2.0$ \\
\hline $\mathrm{S}_{\mathrm{vO}_{2}} \%$ & $>65$ & $60-65$ & $<60$ \\
\hline $\begin{array}{l}\text { Adopted from the "risk tab } \\
\text { pulmonary hypertension [6]. } \\
N \text {-terminal pro-hormone of } \\
\text { cardiac index; } S_{\mathrm{VO}_{2}} \text { : mixed ven }\end{array}$ & $\begin{array}{l}\text { in the } 2015 \text { ESC/ERS } \\
\text { O: World Health Organiz } \\
\text { in natriuretic peptide; } R \\
\text { oxygen saturation. }\end{array}$ & $\begin{array}{l}\text { elines for the diag } \\
\text { ר; 6MWD: 6-min wa } \\
\text { ight atrium; RAP: }\end{array}$ & $\begin{array}{l}\mathrm{S} \text { and treatment of } \\
\text { stance; NT-proBNP: } \\
\text { atrial pressure; } \mathrm{Cl} \text { : }\end{array}$ \\
\hline
\end{tabular}

group were in an exploratory analysis further divided into a low-intermediate (risk score 1.5-1.99) and a high-intermediate (risk score 2.0-2.4) risk group. Patients were also divided into four groups based on the proportion of low-risk variables $(0-24,25-49,50-74$ or $75-100 \%$, respectively), as defined by the ESC/ ERS "risk table" [6]. Risk stratification was performed at diagnosis and during follow-up. SPAHR does not include data on clinical signs of right heart failure, progression of symptoms or syncope, and all variables in the ESC/ERS "risk table" could therefore not be used for risk assessments.

\section{Statistics}

Transplantation-free survival in relation to risk assessments was analysed using Kaplan-Meier estimates and Cox proportional hazards regressions. In multivariate models, a backward stepwise approach was used, and only complete cases were analysed. Risk profile (low or intermediate/high risk), age, sex, BMI, eGFR and comorbidities were used as covariates. BMI, eGFR and comorbidities were included in the multivariate analyses if the p-value was $<0.15$ in univariate analyses. An event was defined as death or lung transplantation. Patients were censored on May 21, 2018. Results are presented as the hazard ratio with 95\% confidence intervals. Survival analyses were performed based on available 3-, 4- and 5-year follow-up data, and survival time was calculated from the respective follow-up. Analyses were performed on the entire study cohort, but also after excluding patients who, at diagnosis, fulfilled one of the three following criteria: 1$) \geqslant 3$ risk factors for heart failure with preserved ejection fraction (HFpEF; i.e. BMI $\geqslant 30 \mathrm{~kg} \cdot \mathrm{m}^{-2}$, diabetes mellitus, hypertension, ischaemic heart disease); 2) atrial fibrillation; or 3) age $>75$ years. The strategy was adopted from the inclusion and exclusion criteria of the TRITON study (NCT02558231). For these latter analyses, BMI, eGFR and comorbidities were not included as covariates in the Cox proportional hazards regressions. Descriptive data are presented as median (interquartile range) for continuous and absolute or per cent for categorical variables. A p-value $<0.05$ was considered statistically significant. Analyses were performed in IBM SPSS Statistics v.25 (IBM, Chicago, IL, USA).

\section{Results}

\section{Study population}

Characteristics at diagnosis and at follow-ups, 3-, 4-, and 5 years post-diagnosis, are shown in tables 2 and 3. The most common PAH subset was IPAH/FPAH (49\%), followed by APAH-CTD (30\%) and APAH-CHD (13\%). Median age at diagnosis was 60 (43-70) years and $68 \%$ of patients were female. $13 \%$ received upfront combination therapy, whereas $54-58 \%$ received sequential combination therapy during follow-up. Median follow-up time was 33 (14-64) months for the 3-year, 33 (13-59) for the 4-year and 35 (19-54) for the 5-year follow-up groups. There were 99 deaths or lung transplantations in the 3-year, 71 in the 4 -year and 44 in the 5-year follow-up groups.

Characteristics of the population after excluding patients who presented at diagnosis with $\geqslant 3$ risk factors for HFpEF, atrial fibrillation and/or age $>75$ years are shown in table 2 and in online supplementary table S1. Characteristics of the excluded patients are shown in online supplementary table S2. Fifty-three patients could not be classified due to incomplete data with regard to comorbidities or BMI. 


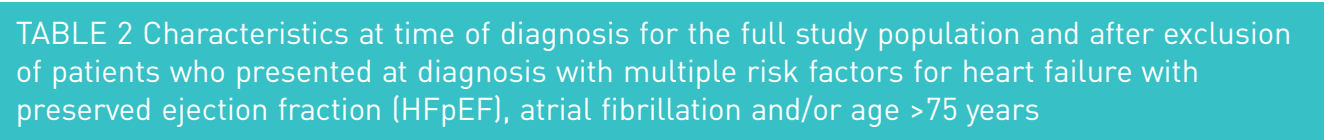

\begin{tabular}{|c|c|c|}
\hline & Full study population & $\begin{array}{c}\text { Study population after exclusion of patients with } \\
\text { multiple risk factors for HFpEF, atrial fibrillation } \\
\text { and/or age }>75 \text { years at diagnosis }\end{array}$ \\
\hline Subjects $\mathrm{n}$ & 386 & 252 \\
\hline Age years & $60(43-70)$ & $53(37-66)$ \\
\hline \multicolumn{3}{|c|}{ WHO functional class $\%$} \\
\hline I & 2 & 2 \\
\hline II & 23 & 26 \\
\hline III & 68 & 64 \\
\hline IV & 7 & 8 \\
\hline 6MWD m & $330(228-445)$ & $373(251-470)$ \\
\hline NT-proBNP ng. L $^{-1}$ & 958 (299-2424) & 803 (230-2087) \\
\hline MPAP $\mathrm{mmHg}$ & $47(38-56)$ & $47(38-57)$ \\
\hline MRAP $\mathrm{mmHg}$ & $6(4-10)$ & $6(3-8)$ \\
\hline PAWP mmHg & $8(6-11)$ & $7(6-10)$ \\
\hline $\mathrm{CO} L \cdot \mathrm{min}^{-1}$ & $4.5(3.6-5.3)$ & 4.5 (3.7-5.3) \\
\hline $\mathrm{CI} L \cdot \min ^{-1} \cdot \mathrm{m}^{-2}$ & $2.5(2.0-3.0)$ & $2.5(2.0-3.1)$ \\
\hline PVR WU & $8.6(5.8-12.0)$ & $8.7(5.7-12.2)$ \\
\hline $\mathrm{S}_{\mathrm{v02}} \%$-units & $65(58-70)$ & $66(60-71)$ \\
\hline $\mathrm{SaO}_{2} \%$-units & 93 (89-95) & $93(89-96)$ \\
\hline MAP mmHg & 95 (85-105) & 95 (85-105) \\
\hline \multicolumn{3}{|c|}{$\begin{array}{l}\text { Data are presented as median linterquartile range), unless otherwise stated. WHO: World Health } \\
\text { Organization; 6MWD: 6-min walk distance; NT-proBNP: N-terminal pro-hormone of brain natriuretic } \\
\text { peptide; MPAP: mean pulmonary arterial pressure; MRAP: mean right atrial pressure; PAWP: pulmonary } \\
\text { arterial wedge pressure; } \mathrm{CO} \text { : cardiac output; } \mathrm{Cl} \text { : cardiac index; PVR: pulmonary vascular resistance; } S_{\mathrm{vo2}} \text { : } \\
\text { mixed venous oxygen saturation; } \mathrm{S}_{\mathrm{a} 02} \text { : arterial oxygen saturation; MAP: mean systemic arterial pressure. }\end{array}$} \\
\hline
\end{tabular}

Risk group distributions at baseline and during follow-up

There were 7 (6-7), 5 (3-5), 4 (3-5) and 4 (3-5) variables available for risk assessments at diagnosis and at the 3-, 4- and 5-year follow-ups, respectively. The frequency by which each variable was used for risk assessment is depicted in online supplementary table S3.

In this population of patients who survived for at least 2.5 years from diagnosis, $31 \%$ were at diagnosis classified as low risk, $62 \%$ as intermediate risk and $7 \%$ as high risk (table 3). At the 3-, 4- and 5-year follow-ups, the corresponding numbers were: 39, 54 and 8\%; 48, 45 and 8\%; and 47, 49 and 4\%, respectively (table 3). Risk group distributions per PAH subset are shown in online supplementary table S4.

After exclusion of patients who presented at diagnosis with $\geqslant 3$ risk factors for HFpEF, atrial fibrillation and/or age $>75$ years, $38 \%$ were at diagnosis classified as low risk, $55 \%$ as intermediate risk and $7 \%$ as high risk. Corresponding numbers at the 3-, 4- and 5-year follow-ups were 47, 47 and 6\%; 58, 37 and 5\%; and 57,38 and 5\%, respectively (online supplementary table S1). Risk group distributions per PAH subset are shown in online supplementary table S5.

Risk group distributions for the excluded patients are shown in online supplementary table S2.

\section{Transplantation-free survival in relation to risk assessments}

In multivariate Cox proportional hazards regressions, a low-risk profile at the 3-, 4- or 5-year follow-ups was an independent predictor of longer transplant-free survival (table 4). Results from the univariate analyses are found in online supplementary table S6. Survival rates differed significantly $(\mathrm{p}<0.001)$ for patients in the low-, intermediate- and high-risk groups at the 3-, 4- and 5-year follow-ups, respectively (figure 1). Survival rates also differed significantly $(\mathrm{p}<0.001)$ based on the proportion of low-risk variables at the 3-, 4- and 5-year follow-ups, respectively (figure 2).

Patients with a high-intermediate risk score had significantly worse survival rates $(\mathrm{p}<0.001)$ than those with a low-intermediate risk score at the 3-, 4- and 5-year follow-ups.

Similar results were found after excluding patients who presented at diagnosis with $\geqslant 3$ risk factors for HFpEF, atrial fibrillation and/or age $>75$ years; see table 5 and online supplementary figures S1 and S2. 
TABLE 3 Additional characteristics at diagnosis and during follow-up for the full study population

\begin{tabular}{|c|c|c|c|c|}
\hline & Diagnosis & $\begin{array}{l}\text { 3-year } \\
\text { follow-up }\end{array}$ & $\begin{array}{l}\text { 4-year } \\
\text { follow-up }\end{array}$ & $\begin{array}{l}\text { 5-year } \\
\text { follow-up }\end{array}$ \\
\hline Subjects $\mathbf{n}$ & 386 & 251 & 193 & 139 \\
\hline Age years & $60(43-70)$ & $60(43-72)$ & $60(42-72)$ & $58(40-71)$ \\
\hline Female sex $\%$ & 68 & 69 & 69 & 71 \\
\hline \multicolumn{5}{|l|}{ PAH subsets $\%$} \\
\hline IPAH/FPAH & 49 & 52 & 51 & 49 \\
\hline APAH-CTD & 30 & 26 & 23 & 22 \\
\hline APAH-CHD & 13 & 13 & 17 & 19 \\
\hline APAH-Others & 9 & 9 & 9 & 10 \\
\hline \multicolumn{5}{|l|}{ Comorbidities \% } \\
\hline Hypertension & 31 & 31 & 25 & 21 \\
\hline Diabetes mellitus & 13 & 13 & 10 & 8 \\
\hline Atrial fibrillation & 9 & 8 & 8 & 8 \\
\hline Previous stroke & 4 & 4 & 2 & 4 \\
\hline Ischaemic heart disease & 10 & 11 & 9 & 7 \\
\hline Thyroid disease & 12 & 14 & 12 & 14 \\
\hline Obesity (BMI $>30 \mathrm{~kg} \cdot \mathrm{m}^{-2}$ ) & 19 & 21 & 15 & 14 \\
\hline $\begin{array}{l}\text { Kidney dysfunction (eGFR } \\
<60 \mathrm{~mL} \cdot \mathrm{kg}^{-1} \cdot \mathrm{m}^{-2} \text { ) }\end{array}$ & 27 & 34 & 26 & 21 \\
\hline \multicolumn{5}{|l|}{ PAH-targeted treatment \% } \\
\hline ERA & 50 & 20 & 23 & 22 \\
\hline PDE5 inhibitor & 20 & 13 & 15 & 12 \\
\hline Prostacyclin & 3 & 1 & 1 & 1 \\
\hline sGC stimulator & 0 & 0 & 0 & 0 \\
\hline Study drug & 3 & 1 & 0 & 0 \\
\hline Dual therapy & 12 & 48 & 43 & 42 \\
\hline Triple therapy & 1 & 10 & 10 & 14 \\
\hline Quadruple therapy & 0 & 0 & 1 & 0 \\
\hline No treatment registered & 12 & 7 & 8 & 7 \\
\hline \multicolumn{5}{|l|}{ Supportive therapy \% } \\
\hline Anticoagulants & 58 & 61 & 64 & 62 \\
\hline Diuretics & 56 & 65 & 59 & 57 \\
\hline Supplemental oxygen & 17 & 26 & 22 & 18 \\
\hline \multicolumn{5}{|l|}{ Risk group \% } \\
\hline Low risk & 31 & 39 & 48 & 47 \\
\hline Intermediate risk & 62 & 54 & 45 & 49 \\
\hline High risk & 7 & 8 & 8 & 4 \\
\hline
\end{tabular}

Data are presented as median (interquartile range), unless otherwise stated. PAH: pulmonary arterial hypertension; APAH: associated pulmonary arterial hypertension; IPAH: idiopathic pulmonary arterial hypertension; FPAH: familial pulmonary arterial hypertension; CTD: connective tissue disease; CHD: congenital heart disease; BMI: body mass index; eGFR: estimated glomerular filtration rate; ERA: endothelin receptor antagonist; PDE5: phosphodiesterase 5; sGC: soluble guanylate cyclase.

TABLE 4 Independent predictors of transplant-free survival during follow-up for the full study population

\begin{tabular}{lccc} 
& 3-year follow-up & 4-year follow-up & 5-year follow-up \\
\hline Subjects $n$ & $158^{\#}$ & $156^{\#}$ & $121^{\#}$ \\
Low risk & $0.27(0.13-0.58)$ & $0.32(0.16-0.65)$ & $0.33(0.14-0.80)$ \\
Age & NS & $1.03(1.01-1.05)$ & $1.03(1.01-1.05)$ \\
Female sex & $0.46(0.26-0.79)$ & NS & NS \\
eGFR & $0.98(0.96-0.99)$ & NS & NS
\end{tabular}

Data are presented as hazard ratio $(95 \% \mathrm{CI})$. During follow-up in the full study cohort there were 56 events in the 3-year follow-up group, 51 in the 4-year follow-up group and 36 in the 5-year follow-up group. NS: not significant; eGFR: estimated glomerular filtration rate. \#: only patients with full coverage of the variables are included in the multivariate analyses. 

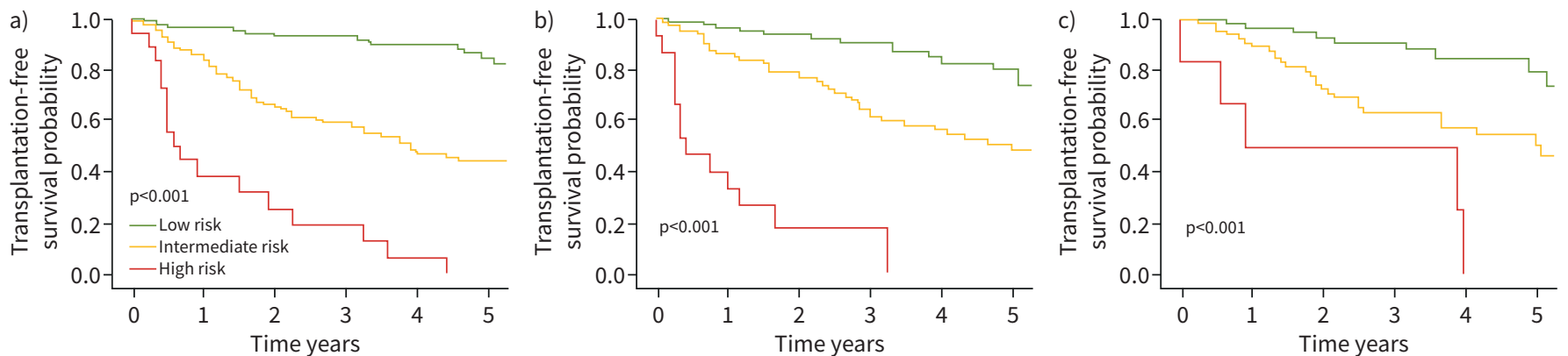

FIGURE 1 Transplant-free survival rates according to risk group at the a) 3-, b) 4- and c) 5-year follow-up, respectively, for the full study population.

\section{Discussion}

The main findings of the present study emphasise that a low-risk profile based on the ESC/ERS guidelines' "risk table" presents as a strong, independent predictor of longer transplant-free survival during long-term follow-up of patients with PAH in a "real-life" clinical setting. In addition, the more low-risk variables a patient presented with during follow-up, the better was the prognosis. In previous studies from the Swedish [7], French [8] and international COMPERA [9] PAH registries, three abbreviated versions of the ESC/ERS "risk table" predicted outcome when applied to findings at the diagnostic check-up and at an early follow-up performed within a maximum of 1-2 years from diagnosis. However, the median time from diagnosis to follow-up was only 3-4 months in the Swedish [7] and French [8] studies. In the present study, using "real-life" clinical data, we found that the risk assessment strategy appears to be applicable also during follow-ups for up to 5 years post diagnosis. Our findings hence suggest that the ESC/ERS "risk table" indeed appears to be a valuable tool for the long-term follow-up of patients with PAH. Our data support and add to those of two recent studies, which found that abbreviated versions of the ESC/ERS "risk table" were, in post hoc analyses, successful in discriminating patients with better or worse outcome in the mixed incident and prevalent, treatment-naïve and previously treated populations of the randomised controlled trials PATENT-1, its open-label extension PATENT-2 [10] and GRIPHON [11]. The present findings are of clinical importance as ESC/ERS guidelines recommend that the risk stratification model should be used frequently and repeatedly at consecutive follow-ups during the course of disease and that a low-risk profile should be considered a treatment goal, an approach based on "expert opinion" [6].

As compared to previous studies from the Swedish [7] and COMPERA [9] PAH registries, which used the "SPAHR equation" for risk group allocation at diagnosis and early follow-up, there was in the present study a greater proportion of patients in the low-risk, and a smaller proportion of patients in the high-risk group, both at baseline and during follow-up. This is likely related to selection bias, as the present study set to investigate the utility of risk assessments during long-term follow-up and included only patients who survived the first years of disease. Nevertheless, those patients who were, based on the risk stratification model, classified as having a high mortality risk at the 3-, 4- or 5-year follow-ups indeed had high mortality rates of $\sim 50-70 \%$ within a year. This suggests that escalation of medical treatment and/or listing for lung transplantation, as well as close clinical monitoring, is to be recommended for patients presenting with a high-risk profile also later in the disease.
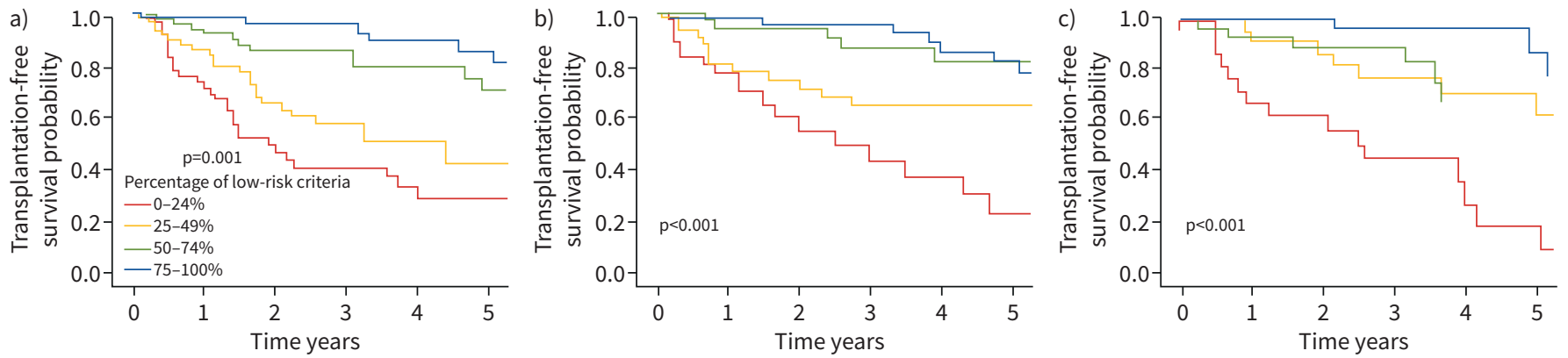

FIGURE 2 Transplant-free survival rates according to the proportion of low-risk variables at the a) 3-, b) 4- and c) 5-year follow-up, respectively, for the full study population. 
TABLE 5 Independent predictors of transplant-free survival during follow-up for the study population after exclusion of patients with multiple risk factors for heart failure with preserved ejection fraction (HFpEF), atrial fibrillation and/or age $>75$ years at diagnosis

\begin{tabular}{|c|c|c|c|}
\hline & 3-year follow-up & 4-year follow-up & 5-year follow-up \\
\hline Subjects $n$ & $167^{\#}$ & $132^{\#}$ & $99^{\#}$ \\
\hline Low risk & $0.24(0.12-0.48)$ & $0.37(0.18-0.75)$ & $0.37(0.15-0.90)$ \\
\hline Age & $1.03(1.01-1.04)$ & $1.03(1.01-1.05)$ & $1.04(1.01-1.07)$ \\
\hline Female sex & $0.51(0.29-0.91)$ & NS & NS \\
\hline \multicolumn{4}{|c|}{$\begin{array}{l}\text { Data are presented as hazard ratio }(95 \% \mathrm{CI}) \text {. During follow-up in the cohort of patients where those who } \\
\text { presented with multiple risk factors for HFpEF, atrial fibrillation and/or age }>75 \text { years at diagnosis had } \\
\text { been excluded, there were } 53 \text { events in the } 3 \text {-year follow-up group, } 36 \text { in the } 4 \text {-year follow-up group and } \\
25 \text { in the } 5 \text {-year follow-up group. Ns: not significant. }{ }^{\#} \text { : only patients with full coverage of the variables are } \\
\text { included in the multivariate analyses. }\end{array}$} \\
\hline
\end{tabular}

Interestingly, in an exploratory analysis we found that patients with a high-intermediate risk score had significantly worse survival rates than those with a low-intermediate risk score. This indicates that the large intermediate risk group should possibly be re-defined to take into account the probable need for more aggressive treatment of subjects with a higher intermediate risk score, although further investigation of this in future studies is still needed. Beside the present approach for subgrouping the intermediate risk group by use of the "SPAHR equation", one may for future studies for instance adjust the cut-offs for the intermediate- and high-risk groups so that high-intermediate risk patients are classified as high risk or create new cut-offs for classification of low-intermediate and high-intermediate risk patients, respectively. The good prognosis of patients in the low-risk group underlines that a low-risk profile indeed is a rational treatment goal.

Recent registry data show that there has been a shift in demographics among PAH patients, with a higher proportion of older, more frequently male patients with a greater comorbidity burden $[1,15]$. Therefore, we chose to perform separate sensitivity analyses after excluding patients who presented at diagnosis with multiple risk factors for HFpEF, atrial fibrillation and/or age $>75$ years, in addition to our main examinations of the full study cohort. At diagnosis, NT-proBNP ( $N$-terminal pro-hormone of brain natriuretic peptide) levels were lower and 6MWD was longer after exclusion of patients who presented at diagnosis with multiple risk factors for HFpEF, atrial fibrillation and/or age $>75$. This may reflect the lower age and a smaller burden or cardiovascular comorbidity in this subgroup even though there were no differences in invasively measured haemodynamic variables at rest. Importantly, a low-risk profile and the proportion of low-risk variables were strong, independent predictors of transplant-free survival also in this group of patients with a diagnosis of $\mathrm{PAH}$, representing patients similar to those previously included in randomised controlled trials.

The group of PAH patients with multiple risk factors for HFpEF, atrial fibrillation and/or age $>75$ years was rather small and was not further analysed with respect to risk assessments or outcome. Patients with such "atypical", but clinically relevant, characteristics have normally not been included in randomised controlled trials, but registry data suggest that they respond less well to PAH-targeted treatments [15-17]. It has been proposed that these patients may represent individuals with misdiagnosed HFpEF with excessive vasoconstriction and/or remodelling or alternatively a new "phenotype" of PAH. We acknowledge the diagnostic difficulties that clinicians encounter when confronted with such patients.

\section{Limitations}

This study encompasses general standard limitations of a multicentre, registry-based observational study, such as lack of a standardised study protocol, missing data and the possibility of selection bias with respect to follow-ups. The patients included in the study were diagnosed during a time-span of $\sim 14$ years, a period when diagnostic criteria, available treatments, treatment strategies and guidelines have varied. As SPAHR does not include data on clinical signs of right heart failure, progression of symptoms or syncope, all variables in the ESC/ERS "risk table" could not be used for risk assessments in the present study. The method for risk assessment was additionally adjusted to fit a retrospective registry-based data set. There were fewer variables available for risk assessment during follow-up than at diagnosis, and we do not know whether this affected the risk group assessment. The study population is, in a wider context, rather small, but in light of PAH being a rare disease, larger study groups are infrequent. There were no specific analyses for different PAH subsets, although separate analyses were performed for patients with or without 
multiple risk factors for HFpEF, atrial fibrillation and/or age $>75$ years at diagnosis. Application of comprehensive risk stratification models to future prospective studies and studies of various PAH subsets are thus warranted, including studies of PAH patients not typically included in clinical trials.

\section{Conclusions}

The results of the present study suggest that the ESC/ERS guidelines' "risk table" for patients with PAH is valid also during long-term follow-up in a "real-life" setting. Our findings endorse the ESC/ERS guidelines' "expert opinion" of using the "risk table" for repeated comprehensive risk evaluations and goal-oriented therapy in order to improve outcome in PAH.

We furthermore encourage future international collaborations that in addition aim to investigate the characteristics, treatment response, risk factors and outcome for the subset of patients with multiple comorbidities and/or high age, as well as for various PAH subsets. Future studies for better characterisation of the intermediate risk group are also warranted.

Acknowledgements: We acknowledge the work of the SPAHR registrars at the Swedish PAH centres and Uppsala Clinical Research Centre for developing and administering the SPAHR platform. We acknowledge the SPAHR steering committee and colleagues involved in the initiation of SPAHR and the Swedish Association for Pulmonary Hypertension (SveFPH).

Support statement: During the initiation of SPAHR, Actelion Pharmaceuticals Sweden AB, Bayer Health Care, Eli Lilly Sweden, GlaxoSmithKline, NordInfu Care and Pfizer gave financial support. Since 2011, SPAHR has qualified as a national quality register and the Swedish Association of Local Authorities and Regions give financial support. Financial supporters had no role in data collection, analysis or interpretation and no right in disapproving the manuscript.

Conflict of interest: D. Kylhammar reports grants from The Swedish Association for Pulmonary Hypertension in cooperation with Bayer Health Care, and lecture fees from Actelion Pharmaceuticals Sweden AB and GlaxoSmithKline, outside the submitted work. C. Hjalmarsson reports grants and personal fees for research advisory boards from Actelion Pharmaceuticals Sweden AB, and grants from MSD, outside the submitted work. R. Hesselstrand has nothing to disclose. K. Jansson has nothing to disclose. M. Kavianipour has nothing to disclose. B. Kjellström has nothing to disclose. M. Nisell reports lecture and consultation fees from Actelion Pharmaceuticals Sweden AB, Pfizer, Bayer HealthCare, NordicInfu Care and GlaxoSmithKline outside the submitted work. S. Söderberg reports grants and lecture fees from Actelion Pharmaceuticals Sweden AB, and grants from Pfizer, outside the submitted work. G. Rådegran reports grants, lecture fees and research advisory boards from Actelion Pharmaceuticals Sweden $A B$ and GlaxoSmithKline; lecture fees and research advisory boards from Bayer Health Care; lecture fees from Nordic InfuCare and Sandoz/Novartis; and research advisory boards for Acceleron, Arena, Eli Lilly and Sanofi-Aventis, all outside the submitted work.

\section{References}

1 Rådegran G, Kjellström B, Ekmehag B, et al. Characteristics and survival of adult Swedish PAH and CTEPH patients 2000-2014. Scand Cardiovasc J 2016; 50: 243-250.

2 Humbert M, Sitbon O, Yaïci A, et al. Survival in incident and prevalent cohorts of patients with pulmonary arterial hypertension. Eur Respir J 2010; 36: 549-555.

3 Benza RL, Miller DP, Barst RJ, et al. An evaluation of long-term survival from time of diagnosis in pulmonary arterial hypertension from the REVEAL registry. Chest 2012; 142: 448-456.

4 Simonneau G, Gatzoulis MA, Adatia I, et al. Updated clinical classification of pulmonary hypertension. J Am Coll Cardiol 2013; 62: Suppl. 25, D34-D41.

5 Simonneau G, Montani D, Celermajer DS, et al. Haemodynamic definitions and updated clinical classification of pulmonary hypertension. Eur Respir J 2019; 53: 1801913.

6 Galiè N, Humbert M, Vachiéry JL, et al. 2015 ESC/ERS Guidelines for the diagnosis and treatment of pulmonary hypertension. Eur Respir J 2015; 46: 903-975.

7 Kylhammar D, Kjellström B, Hjalmarsson C, et al. A comprehensive risk stratification at early follow-up determines prognosis in pulmonary arterial hypertension. Eur Heart J 2018; 39: 4175-4181.

8 Boucly A, Weatherald J, Savale L, et al. Risk assessment, prognosis and guideline implementation in pulmonary arterial hypertension. Eur Respir J 2017; 50: 1700889.

9 Hoeper MM, Kramer T, Pan Z, et al. Mortality in pulmonary arterial hypertension: prediction by the 2015 European pulmonary hypertension guidelines risk stratification model. Eur Respir J 2017; 50: 1700740.

10 Humbert M, Farber HW, Ghofrani HA, et al. Risk assessment in pulmonary arterial hypertension and chronic thromboembolic pulmonary hypertension. Eur Respir J 2019; 53: 1802004.

11 Sitbon O, Chin KM, Channick RN, et al. Risk assessment in pulmonary arterial hypertension: insights from the GRIPHON study. J Heart Lung Transplant 2020; 39: 300-309.

12 Galiè N, Torbicki A, Barst R, et al. Guidelines on diagnosis and treatment of pulmonary arterial hypertension. The Task Force on Diagnosis and Treatment of Pulmonary Arterial Hypertension of the European Society of Cardiology. Eur Heart J 2004; 25: 2243-2278.

13 Galiè N, Hoeper MM, Humbert M, et al. Guidelines for the diagnosis and treatment of pulmonary hypertension: the Task Force for the Diagnosis and Treatment of Pulmonary Hypertension of the European Society of Cardiology (ESC) and the European Respiratory Society (ERS), endorsed by the International Society of Heart and Lung Transplantation (ISHLT). Eur Heart J 2009; 30: 2493-2537.

14 Cockcroft DW, Gault MH. Prediction of creatinine clearance from serum creatinine. Nephron 1976; 16: 31-41.

15 Hoeper MM, Huscher D, Ghofrani HA, et al. Elderly patients diagnosed with idiopathic pulmonary arterial hypertension: results from the COMPERA registry. Int J Cardiol 2013; 168: 871-880. 
16 Hjalmarsson C, Rådegran G, Kylhammar D, et al. Impact of age and comorbidity on risk stratification in idiopathic pulmonary arterial hypertension. Eur Respir J 2018; 41: 1702310.

17 Opitz C, Hoeper MM, Gibbs JS, et al. Pre-capillary, combined, and post-capillary pulmonary hypertension: a pathophysiological continuum. J Am Coll Cardiol 2016; 68: 368-378. 